\title{
Multiple Reference Frames in Cortical Oscillatory Activity during Tactile Remapping for Saccades
}

\author{
Verena N. Buchholz, Ole Jensen, and W. Pieter Medendorp \\ Radboud University Nijmegen, Donders Institute for Brain, Cognition and Behaviour, NL 6500 HE, Nijmegen, The Netherlands
}

Single-unit recordings have shown that the brain uses multiple reference frames in spatial processing. The brain could use this neural architecture to implicitly create multiple modes of representation at the population level, with each reference frame weighted as a function of task demands. Using magnetoencephalography, we tested this hypothesis by studying the reference frames in rhythmic neuronal synchronization - a population measure - during tactile remapping for saccades. Human subjects fixated either to the left or right of the body midline, while a tactile stimulus was applied to an invisible fingertip, located either left or right of fixation. After a variable delay, they looked at the remembered stimulus location. Results show a transient body-centered, stimulus-induced gammaband response $(70-90 \mathrm{~Hz})$ in somatosensory areas, contralateral to the stimulated hand. Concurrently, a gamma-band response occurred in posterior parietal cortex (PPC), contralateral to the gaze-centered location of the stimulus, even though the stimulus was not seen. The temporal overlap of these early representations suggests that there is a fast bottom-up sensory-induced remapping in PPC, taking into account the relative positions of eyes and hand. The gaze-centered representation in PPC was sustained in a high gamma range (85-115 $\mathrm{Hz}$ ) and increased in power closer to the initiation of the saccade. Lower-frequency rhythms (alpha, beta) showed body-centered power modulations in somatosensory areas in anticipation of the stimulus and a mixture of reference frames in PPC after stimulus presentation. We conclude that oscillatory activity reflects the time-varying coding of information in body- and gaze-centered reference frames during tactile remapping for saccades.

\section{Introduction}

The brain uses topographic maps in the early stages of cortical sensory processing. For example, processing of visual stimuli takes place in retinotopic maps; the initial processing of touch occurs in somatotopic maps. Higher-order association areas, such as the posterior parietal cortex (PPC), further process this spatial information for control of various cognitive functions including sensory integration, spatial attention, and motor planning (Andersen and Cui, 2009).

Monkey single-unit recordings have shown that the processing in the PPC involves a mixture of reference frames, including gaze-centered, body-centered, and intermediate coordinates (Stricanne et al., 1996; Avillac et al., 2005; Mullette-Gillman et al., 2005; Chang et al., 2009; Mullette-Gillman et al., 2009; Chang and Snyder, 2010). Recent models suggest that this neural architecture provides the PPC with a mechanism to implicitly create multiple modes of representation at the population level, with each reference frame weighted as a function of global task demands (Pouget et al., 2002; McGuire and Sabes, 2009).

In support, Bernier and Grafton (2010) reported that PPC switches the dominant reference frame in different sensory con-

Received July 5, 2011; revised Aug. 30, 2011; accepted Sept. 28, 2011.

Author contributions: V.N.B. and W.P.M. designed research; V.N.B. performed research; 0 .J. contributed unpublished reagents/analytic tools; V.N.B., 0.J., and W.P.M. analyzed data; V.N.B. and W.P.M. wrote the paper.

Correspondence should be addressed to Verena N. Buchholz, Buchholz Radboud University Nijmegen, Donders Institute for Brain, Cognition and Behaviour, Centre for Cognition, P.0. Box 9104, NL-6500 HE, Nijmegen, The Netherlands. E-mail:verena.buchholz@donders.ru.nl.

DOI:10.1523/JNEUROSCI.3404-11.2011

Copyright $\odot 2011$ the authors $\quad 0270-6474 / 11 / 3116864-08 \$ 15.00 / 0$ texts. Using fMRI, which assesses the activity of larger neuronal populations, they found a merely gaze-centered code for reaches to visual targets and a body-centered code for proprioceptive reaches in precuneus areas. Yet, despite these important insights, the question remains unanswered of how neuronal populations in spatial maps can flexibly determine their reference frame.

Rhythmic neuronal synchronization has been suggested as a prime mechanism to bias competition between multiple representations in a neuronal population. In particular, highfrequency oscillations in the gamma band $(>30 \mathrm{~Hz})$ have been shown to amplify behaviorally relevant representations (Womelsdorf and Fries, 2007; Fries et al., 2008). Taking this a step further, it can be hypothesized that gamma-band rhythms could also selectively emphasize information in particular reference frames, but this is not known. Lower-frequency rhythms (alpha: $10 \mathrm{~Hz}$; beta: $18-30 \mathrm{~Hz}$ ), generally regarded as a gating mechanism, are proposed to support the selective processing in the gamma band (Thut et al., 2006; Medendorp et al., 2007; Jensen and Mazaheri, 2010).

Recent local field potential and magnetoencephalographic (MEG) recordings of the PPC indicate contralateral spatial selectivity in these frequency bands during the planning of saccades to memorized visual targets (Pesaran et al., 2002; Van Der Werf et al., 2008). But because this task imposes a fixed (gaze-centered) relationship between sensory and motor coordinates, it cannot identify any coexisting reference frames at the population level during the sensorimotor transformation.

To address this question, we used magnetoencephalography to study the reference frames in synchronized population activity 
during a more complex sensorimotor mapping: saccades to tactile targets delivered to the hand. By varying the location of the target in either body or gaze coordinates, we tested in which reference frames the rhythms in somatosensory cortex (S1), PPC, and other association areas operate during tactile remapping for saccades. We report that tactile remapping is supported by several low- and high-frequency rhythms, which show spatial selectivity in both reference frames, but with different time courses across the parietal network.

\section{Materials and Methods}

Participants. Twenty subjects (age range, 23-47 years; 5 female; 3 lefthanded), free of any known sensory, perceptual, or motor disorders, volunteered to participate in the experiment. All subjects provided written informed consent according to institutional guidelines of the local ethics committee (Committee on Research Involving Human Subjects, region Arnhem-Nijmegen, the Netherlands).

Setup. Participants sat in the MEG system that was placed in a magnetically shielded room. Their hands and elbows were placed on a stimulus device, shown in Figure $1 \mathrm{~A}$, which was located at a distance of $32 \mathrm{~cm}$ in front of the body. The stimulus device was equipped with a set of fiber optic lights (Omron e3x-na) and piezoelectric Braille stimulators (Metec). Subjects viewed the center of the device with a comfortable, slightly downward gaze direction. The device contained one central optical fiber light, and 10 peripheral optic fiber lights, 5 on either side, at horizontal eccentricities of $8,12,18,24$, and $30^{\circ}$. During the actual experiment, which took place in darkness, only those at $18^{\circ}$ eccentricity served as visual fixation points (luminance, $1.2 \mathrm{~cd} / \mathrm{m} 2$ ). Hands were placed on the device such that the four fingertips of each hand, except that of the thumb, were aligned with, but just underneath, the nearest four peripheral lights, as depicted in Figure $1 \mathrm{~A}$. Each of these fingertips overlaid a piezoelectric Braille stimulation pin. A tactile stimulus was presented to the fingertip by transiently raising this pin with an amplitude of $\sim 2 \mathrm{~mm}$, holding it elevated for $20 \mathrm{~ms}$, and then lowering it again. The hands were blocked from vision. Auditory white noise was presented to the subjects through pneumatic earphones to mask the sound generated by the Braille stimulation. The low currents necessary for driving piezoelectrical stimulation created short-lived artifacts in the MEG signals. Visual and tactile stimuli were controlled using Presentation 12.2 software (Neurobehavioral Systems).

MEG data were recorded continuously using a whole head system with 275 axial gradiometers (Omega 2000, CTF Systems). Head position with respect to the sensor array was measured using localization coils fixed at anatomical landmarks (nasion, and left and right ear). Horizontal and vertical electro-oculograms (EOGs) were recorded using electrodes placed below and above the left eye, and at the bilateral outer canthi. Impedance of all electrodes was kept at $<5 \mathrm{k} \Omega$. During the experiment, eye recordings were continuously inspected to ensure the subject was vigilant and performed the task correctly. MEG and EOG signals were low-pass filtered at $300 \mathrm{~Hz}$, sampled at $1200 \mathrm{~Hz}$, and then saved to disk.

Structural full-brain MRIs were acquired with a $1.5 \mathrm{~T}$ Siemens Sonata scanner using a standard T1-weighted scan sequence (FA $=15^{\circ}$; voxel size, $1.0 \mathrm{~mm}$ in-plane; $256 \times 256 ; 164$ slices; $\mathrm{TR}=760 \mathrm{~ms}$; $\mathrm{TE}=5.3 \mathrm{~ms}$ ). These anatomical MRIs were recorded with anatomical reference markers at the same locations as the head position coils during the MEG recordings. The reference markers allow alignment of the MEG and MRI coordinate systems, such that the MEG data can be related to the anatomical structures within the brain.

Experimental paradigm. Subjects performed a delayed-saccade task with tactile stimuli (see Fig. $1 A$ ). Each trial began with the simultaneous presentation of two brief tactile pulses spatially congruent with the fixation light at the location of the tip of the ring finger of the right or the left hand. This initial presentation of spatially congruent visual and tactile stimuli was used to prevent drift in the proprioceptively sensed position of the unseen hand in the dark. Subjects were instructed to fixate at the light.

Then, as shown in Figure $1 A$, after a baseline period of $1500 \mathrm{~ms}$, the target was presented by means of a tactile stimulus to either the index finger or the little finger of the same hand while fixating (green or blue circles). This was followed by a memory delay with a random duration between 1500 and $2000 \mathrm{~ms}$, during which the subject maintained fixation. Subsequently, the fixation light was turned off, instructing the subject to saccade toward the remembered location of the tactile target. Then, $800 \mathrm{~ms}$ later, the next trial started by turning on the fixation light. To ensure a constant vigilance of the subjects throughout the experiment, we also included trials (5\%) with a shorter delay period $(800 \mathrm{~ms})$, although these trials were not further analyzed. Figure $1 A$ illustrates the two conditions that involve right-hand stimulation, with the stimulated finger located either to the left or right of fixation. Thus, the hand of stimulation was predictable on the basis of the fixation, whereas the gaze-centered direction (i.e., visual hemifield) of tactile stimulation could not be anticipated.

The paradigm had four different conditions regarding the location of the target, which were organized into a $2 \times 2$ design, with side (left vs right) and reference frame (gaze vs body) as factors (see Fig. $1 B$ ). That is, the location of the tactile target could be represented as left (hand) or right (hand) to the body, the difference in spatial tuning probing bodycentered lateralization. The location of the tactile target could also be represented as left or right (side) from the gaze line, the difference defining gaze-centered lateralization. The relationship between the side of the target in the body frame and the side of the same target in the gaze frame is not fixed, but depends on the location of the hand relative to gaze. As a result, in conditions 1 (left relative to gaze/right relative to body) and 4 (right relative to gaze/left relative to body), the representation of the stimulus should be lateralized to different hemispheres when encoded in gaze- and body-centered reference frames, while in conditions 2 (right relative to gaze/right relative to body) and 3 (left relative to gaze/left relative to body) lateralization in both references should be to the same hemisphere. Thus, by exploiting hemispheric lateralization, we can distinguish gaze- and body-centered reference frames in the regions that are involved in tactile remapping for saccades.

Subjects performed eight blocks of 90 trials each, in which target locations were pseudorandomly interleaved. Each trial lasted for 3800-4300 ms. A brief rest was provided between the blocks during which the subjects could move their hands and eyes freely.

Behavioral analysis. Trials in which participants broke fixation, blinked during a trial, or generated saccades before the go-cue were excluded. Also, trials in which the participant did not saccade into the correct direction were discarded from further analysis. On average, $533 \pm 22($ mean \pm SE) trials per participant were accepted for further analysis. The number of rejected trials did not differ between conditions (ANOVA, $\left.F_{(3,76)}=0.18, p>0.10\right)$. Mean ( \pm SE) saccadic reaction time across the four conditions was $266 \pm 21 \mathrm{~ms}$. Reaction times did not differ among conditions (ANOVA, $F_{(3,76)}=0.28, p>0.10$ ).

MEG data analysis. Open-source Fieldtrip software (http://fieldtrip. fcdonders.nl/) was used to analyze the MEG data. A planar gradient estimate was calculated from the axial gradiometer signals using the signals from neighboring sensors (Bastiaansen and Knösche, 2000). This simplifies the interpretation of the sensor-level data, as the maximal signal is located above the source with planar gradients (Hämäläinen et al., 1993). The sum of the calculated horizontal and vertical planar MEG field gradients was computed to obtain the power at each virtual planar gradiometer location.

Time-frequency representations (TFRs) were computed based on a Fourier approach, applying a sliding window, with neighboring time points temporally segregated by $50 \mathrm{~ms}$. To optimize time-frequency resolution, we analyzed separately two frequency ranges (2-30 and $30-100 \mathrm{~Hz}$ ). For the lower frequencies, we used a sliding window of $500 \mathrm{~ms}$ and a Hanning taper, resulting in a spectral smoothing of $\sim 3$ $\mathrm{Hz}$. For the higher frequencies $(30-100 \mathrm{~Hz})$, we applied a multitaper approach (Percival and Walden, 1993) using a sliding window of $400 \mathrm{~ms}$ and 11 orthogonal Slepian tapers. This resulted in a spectral smoothing of $\sim 14 \mathrm{~Hz}$.

The piezoelectrical stimulation induced short-lived artifacts in the MEG signals. Figure $1 C$ demonstrates these artifacts for the four stimulus conditions in a time-domain analysis of event-related fields observed at left and right central sensors (covering S1), during the first $500 \mathrm{~ms}$ after 
stimulus onset. The presentation of the stimulus, by the two brief successive piezoelectric pulses, caused artifacts between times 0 and $50 \mathrm{~ms}$. From 50 ms onward, the event-related field amplitudes indicate a clear bias for targets presented to the contralateral hand, as expected, independent of artifact size. Thus, the artifacts have disappeared $50 \mathrm{~ms}$ after trial onset. However, due to temporal smoothing, their effects could bleed into the frequency analysis up to windows centered at $250 \mathrm{~ms}$ after stimulation. We therefore consider our spectral analyses with analysis windows centered from $300 \mathrm{~ms}$ poststimulus and onward as artifact free.

Based on data from similar tasks, but in the visual domain (Medendorp et al., 2007; Van Der Werf et al., 2008, 2010), we predefined the sensory response interval for the higher frequencies to a $400 \mathrm{~ms}$ window centered at $300 \mathrm{~ms}$ after stimulus presentation and for the lowerfrequency bands to a $500 \mathrm{~ms}$ window centered at $400 \mathrm{~ms}$ after the stimulus. Frequency bands of interest were defined as follows: alpha $10 \mathrm{~Hz}$; beta $18-30 \mathrm{~Hz}$; low gamma $30-60 \mathrm{~Hz}$; and high gamma $60-100 \mathrm{~Hz}$. Each predefined time-frequency tile entered our subsequent statistical analysis at the sensor level. Sustained effects during the late delay period were tested in the $1000 \mathrm{~ms}$ interval before the go-cue, with spectral analysis windows centered from 800 to $200 \mathrm{~ms}$ before the go. Processing of the go-cue and contamination of the motor response during this late delayed period is absent, given the size of the analysis window and the saccade latencies $>250 \mathrm{~ms}$ (see above, Behavioral analysis).

To localize the neural sources of the spectral components of interest, we applied an adaptive spatial filtering (or beamforming) technique (Dynamic Imaging of Coherent Sources) (Gross et al., 2001; Liljeström et al., 2005). Each participant's brain volume was divided into an individually spaced three-dimensional grid using SPM2 (http://www.fil.ion.ucl. ac.uk/spm), with each location corresponding to a location in the regular 1 $\mathrm{cm}$ grid based on a brain template [International Consortium for Brain Mapping; Montreal Neurological Institute (MNI), Montreal, QC, Canada]. We then warped every subject's MRI to fit this template MRI and the template's grid. We subsequently warped the grid back to fit every subject's original MRI to obtain a grid in MNI coordinates for each subject. This procedure allowed us to directly compare grid points across subjects in MNI space without the need to normalize. For each subject, the filter was computed from forward models with respect to dipolar sources at each individual grid point (the leadfield matrix) and the cross-spectral density between all combinations of sensors at the frequency of interest (Nolte, 2003). The computed spatial filter fully passes activity from the location of interest (the individual grid points), while attenuating activity from all other locations (Van Veen et al., 1997). We used single-sphere head models from each subject's individual MRI to calculate the lead field matrix (Nolte, 2003). For every single subject, the source power was estimated relative to the same baseline interval that was used for the corresponding sensor-level analysis. The source reconstruction was tuned to peak power values in the same time interval.

Task-related power and statistical inferences. We computed the taskrelated changes in power in various frequency bands relative to average power in the baseline period (see Fig. $1 \mathrm{~A}$ ). The baseline power was computed over a $400 \mathrm{~ms}$ (higher frequencies) or $500 \mathrm{~ms}$ (lower frequencies) time window centered $300 \mathrm{~ms}$ before the presentation of the stimulus. For each condition, we expressed the difference in log power between the delay period and the baseline as a $t$-score, separately for each subject. The resulting $t$-scores were transformed into $z$-scores as in Medendorp et al. (2007) to obtain normalized estimates of power differences. The resulting $z$-scores, which are well normalized for intrasubject variance, were pooled across subjects (zgroup $=1 / \sqrt{ } N \sum z i$ with $z i$ being the $z$-score of the $i$ th subject). These $z$-values cannot be interpreted as a statistical test outcome, but serve as inputs for the group-level analysis. At the group level, statistical significance of the power modulations was tested at the sensor level by using a nonparametric clustering procedure, which controls for multiple comparisons in time, sensor, and frequency space (for further details about this approach, see Van Der Werf et al., 2008; Van Der Werf et al., 2010).

Body- and gaze-centered selectivity in various frequency bands was assessed by comparing spectral power in conditions with different hands being stimulated and in conditions in which the tactile target is located at opposite directions from the gaze line, respectively. We contrasted the

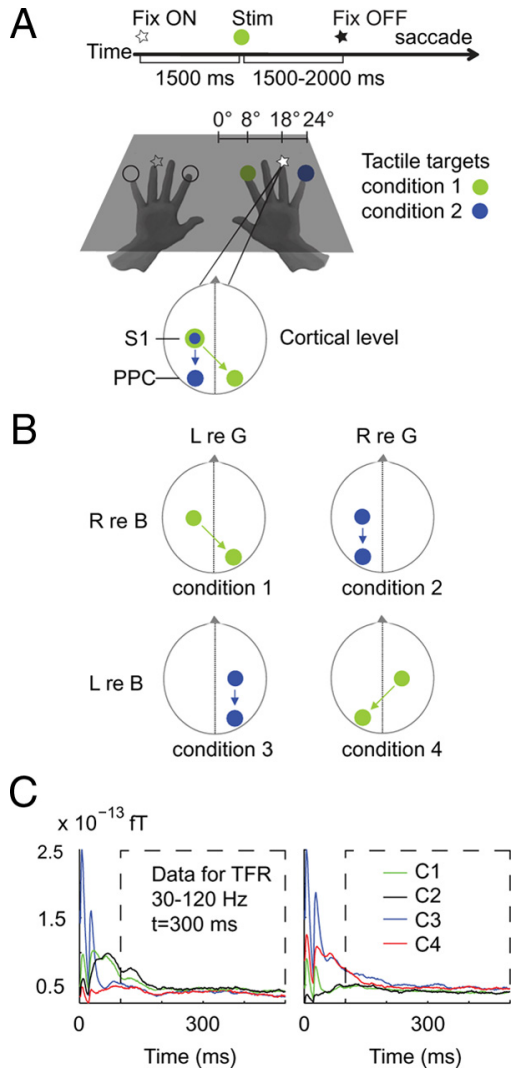

Figure 1. Experimental design. $A$, Sequence of stimuli and subject instructions. A trial began with fixating a light at the position of the tip of the ring finger of the right or left hand. After a baseline period of $1500 \mathrm{~ms}$, the tactile target was presented to either the index or little finger of the same hand. After a further delay of $1500-2000 \mathrm{~ms}$, the visual fixation light was switched off, instructing the subject to make a saccade to the remembered location of the tactile target. $\boldsymbol{B}$, Target locations were based on a $2 \times 2$ factorial design, with factors side (left vs right) and reference frame (gaze vs body). Body-centered lateralization characterizes the power differences for stimuli delivered to the contralateral versus ipsilateral hand. Gaze-centered selectivity compares the power for invisible tactile targets in the contralateral versus ipsilateral visual field. C, Event-related fields at sensors overlaying the left-hand and right-hand somatosensory areas. Colors signify the four conditions ( $(1$ to (4). Stimulus artifacts have disappeared $50 \mathrm{~ms}$ after trial onset. Because of the spectral analysis windows, only time-frequency points from $300 \mathrm{~ms}$ poststimulus and onward are artifact free.

power changes for contralateral versus ipsilateral target locations (defined in either of the two reference frames) for each hemisphere (see Fig. $1 B$ ), and then pooled these hemispheric results to determine the bodyand gaze-centered laterality across hemispheres. Significant sensor clusters were determined for each of these contrasts, based on the predefined time-frequency tiles (see above, MEG data analysis). Statistical analysis of the late delay period was performed for these sensor clusters.

\section{Results}

We investigated the gaze- and body-centered selectivity of power in various frequency bands during tactile remapping. Targets were delivered to either the left or right hand, to finger tips located in either the left or right visual hemifield (Fig. 1). Body-centered lateralization characterizes the power differences for stimuli delivered to the contralateral versus ipsilateral hand. Gaze-centered lateralization compares the power for invisible tactile targets in the contralateral versus ipsilateral visual field.

\section{Higher-frequency activity shows instant sensory remapping} We first present the results of the higher frequencies. The lefthand panels of Figure 2, $A$ and $B$, show the scalp topographies of the body-centered and gaze-centered selectivity of power in the 


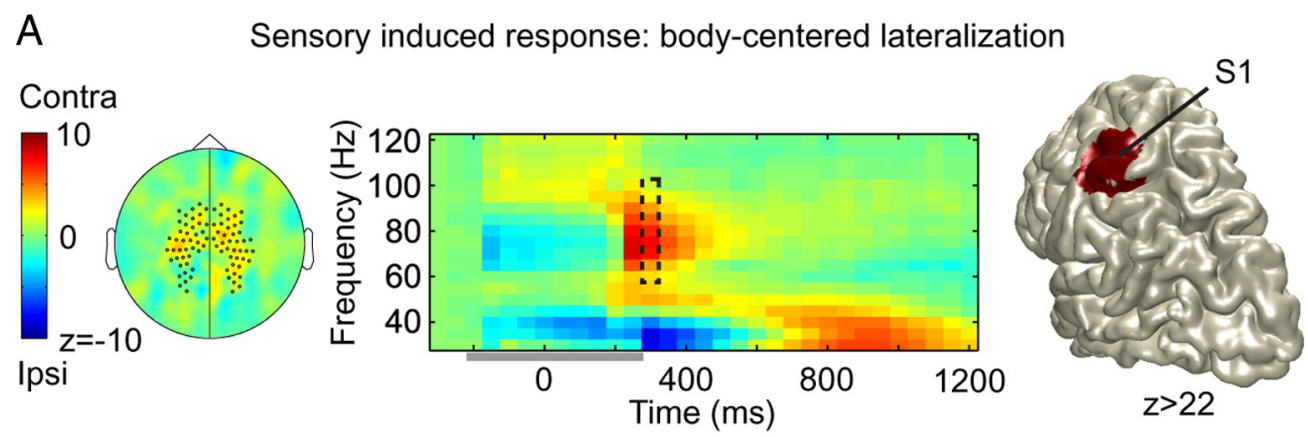

\section{B Sensory induced response: gaze-centered lateralization}
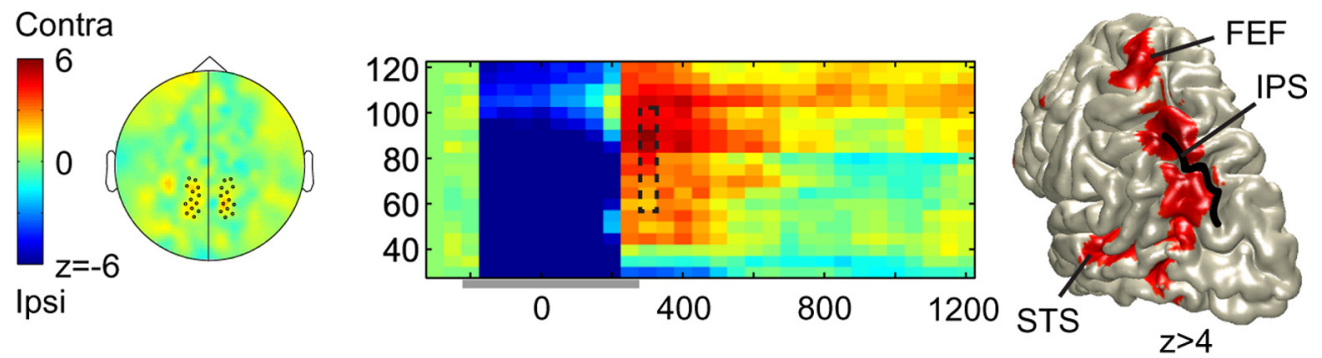

C

Late delay activity: gaze-centered lateralization
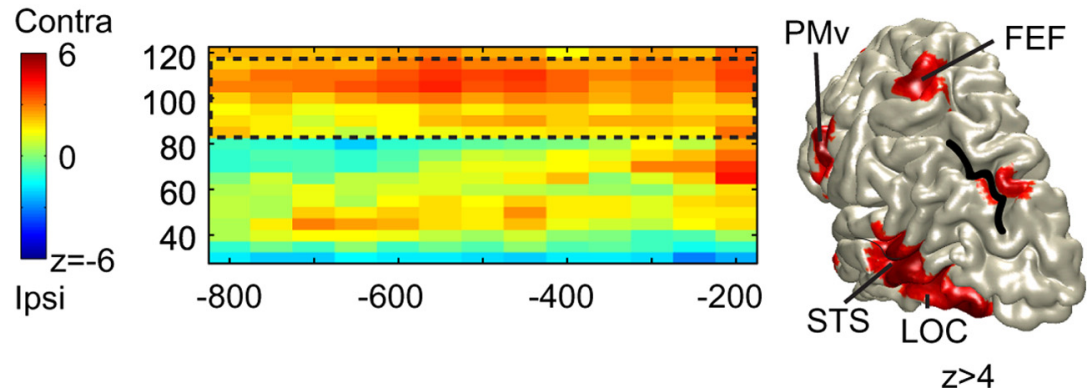

Figure 2. A, Sensory remapping in the gamma band. $A$, Left, Scalp topography of the body-centered gamma-band lateralization $(60-100 \mathrm{~Hz})$ during the sensory response period $(t=300 \mathrm{~ms})$. Significant sensors are marked. Middle, TFR (artifact free from $t=300 \mathrm{~ms}$ onward; see Materials and Methods) of power for the marked sensors, pooled across hemispheres. Time window of artifact-contaminated data indicated by a horizontal gray bar. Right, Source reconstruction of the body-centered gamma-band activity during the sensory response period ( $70-90 \mathrm{~Hz} ; t=300 \mathrm{~ms}$ ). Color format for all panels: warmer (red) colors, increase for targets to contralateral hand; cooler (blue) colors, increase for ipsilateral targets. $\boldsymbol{B}$, The transient gaze-centered gamma band. Left, Topography of gaze-centered gamma-band power $(60-100 \mathrm{~Hz} ; t=300 \mathrm{~ms})$ during the sensory response period. Middle, TFR of marked (significant) sensors. Right, Source reconstruction of the sensory gamma-band response (95-105 Hz; $t=300 \mathrm{ms)}$. Color format for all panels: warmer (red) colors, increase for tactile targets in contralateral visual field; cooler (blue) colors, increase for ipsilateral targets. Artifact window, gray horizontal bar. C, Sustained gaze-centered gamma band, aligned to the go-cue of the saccade. TFR, Sustained power increase at $85-115 \mathrm{~Hz}$. Right, Source reconstruction of the sustained gamma-band activation during the delay ( $95-105 \mathrm{~Hz} ; t=200 \mathrm{~ms}$ before the go-cue). LOC, Lateral occipital cortex.

60-100 Hz gamma band, respectively, in response to the presentation of the tactile target $(300 \mathrm{~ms})$. Data are expressed as $z$-scores pooled across subjects; significant sensors $(p<0.05)$ are marked by dots. Contralateral increases are color coded in warmer (red) hues; ipsilateral increases are color coded using the cooler (blue) tints. As shown, a cluster of sensors overlaying central regions demonstrates body-centered selectivity (Fig. 2A), while a set of posterior sensors shows clear gaze-centered selectivity (Fig. $2 \mathrm{~B}$ ). This suggests that the target, detected and initially processed in body-centered, somatotopic coordinates, is readily remapped into a gaze-centered representation during the sensory response period.

The middle panels of Figure 2, $A$ and $B$, illustrate the timefrequency representations of the two sensor groups in the higher frequencies $(30-120 \mathrm{~Hz})$, aligned to stimulus onset. More specifically, these spectrograms show the combined hemispherespecific changes of power for contralateral versus ipsilateral targets in body-centered and gaze-centered reference frames, re- spectively (for further explanation, see Fig. $1 B$ ). Stimulus artifacts are seen during the first $300 \mathrm{~ms}$ after stimulus onset (for more details, see Materials and Methods and Fig. 1C); the rest of the data from 300 to $1200 \mathrm{~ms}$ into the delay are clean and artifact free. As shown, body-centered lateralization, observed at the central sensors in the 70-90 $\mathrm{Hz}$ range, shows an increase in gamma-band power after stimulus presentation biased to the contralateral hand. This gamma-band activity disappears during the delay period. Along with the occurrence of the transient body-centered response, there is a significant broad-banded gaze-centered bias at the posterior sensors (Fig. $2 \mathrm{~B}$ ). More specifically, the gamma-band activity is higher for targets in the contralateral than in the ipsilateral visual hemifield. This suggests that the initial representation of the target, as sensed in somatotopic, body-centered coordinates, is quite readily remapped during the sensory induced response into retinotopic, gaze-centered coordinates. This gaze-centered representation is kept on-line in a higher frequency range $(85-115 \mathrm{~Hz})$ during the late delay interval, when subjects are awaiting the 


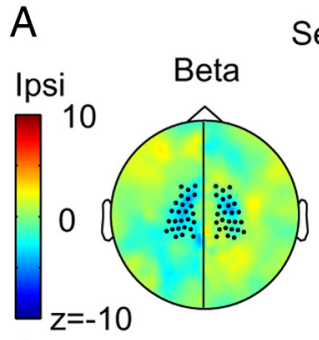

Contra
Sensory induced response: body-c. lateralization

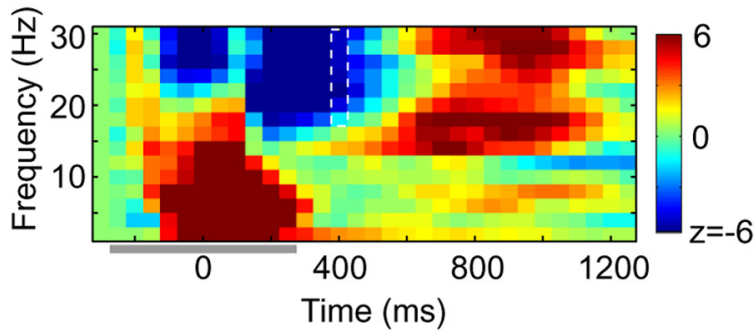

Sensory induced response: gaze-c. lateralization

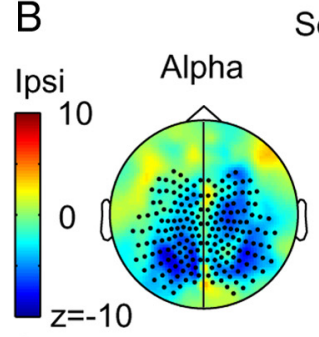

Contra

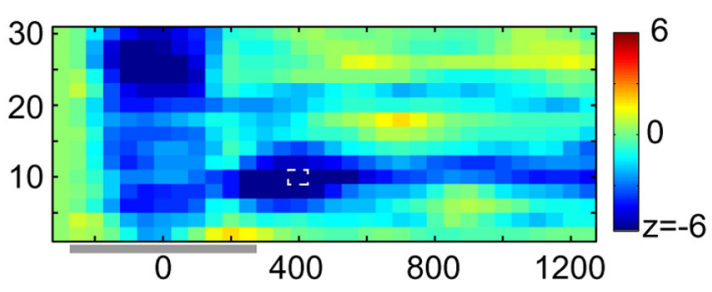

Figure 3. Body-centered beta and gaze-centered alpha rhythms share a common substrate. $\boldsymbol{A}$, Body-centered lateralization. Color format for all panels: warmer (red) colors, increase for targets to contralateral hand; cooler (blue) colors, increase for ipsilateral targets. Left, Scalp topography shows a beta power decrease $(18-30 \mathrm{~Hz})$ at central sensors (marked) during the sensory response period $(t=400 \mathrm{~ms}$ ). Middle, Time-frequency resolved power changes for the marked sensors. Artifactual data indicated by a horizontal gray bar. Right, Top, Source reconstruction of the body-centered sensory response ( $18-30 \mathrm{~Hz} ; t=400 \mathrm{~ms})$. Right, Bottom, Source reconstruction of the beta rebound during the delay period ( $t=800 \mathrm{~ms})$. $\boldsymbol{B}$, Gaze-centered activity. Color format for all panels: warmer (red) colors, increase for targets in contralateral field; cooler (blue) colors, increase for ipsilateral targets. Left, Scalp topography shows an alpha power decrease ( $10 \mathrm{~Hz}$; $t=$ 400) at posterior and central sensors (marked). Middle, Time-frequency resolved power changes for the marked sensors. Right, Source reconstruction of the gaze-centered alpha-band suppression during the sensory response $(10 \mathrm{~Hz} ; t=400 \mathrm{~ms})$. Body-centered beta and gaze-centered alpha-band power share a common substrate in the SPL.

go-cue to make the saccade. In support of this interpretation, when the data are aligned to the onset of the go-cue, the sustained gamma response clearly builds up in strength closer to movement execution (Fig. $2 C)$. Lower gamma-band activity $(30-60 \mathrm{~Hz})$ did not show any significant modulation $(p>0.05)$. Note that the gamma-band modulations at $\sim 30-40 \mathrm{~Hz}$ reflect spillover effects of the lower-frequency bands, which will be discussed below.

The right-hand panels of Figure 2 show the source reconstructions of the body-centered and gaze-centered power changes in the gamma band, in the same color format. The body-centered power modulations originate from the primary somatosensory cortex (Fig. 2A), specifically from the hand area of the postcentral gyrus. Touch-induced gaze-centered selectivity was localized in several areas. The early gaze-centered selectivity originated from the intraparietal sulcus (IPS) and from an area at the junction of the frontal and precentral sulcus, possibly the frontal eye fields (FEFs), and therefore assigned as FEF (Russo and Bruce, 1994; Van Pelt et al., 2010). Also the superior temporal sulcus (STS) showed an instant gaze-centered selectivity of power. The sustained gamma-band activity during the delay interval was located in the same regions, as well as in lateral occipital and ventral premotor (PMv) regions (Fig. 2C).

Body-centered beta and gaze-centered alpha rhythms share a common substrate in superior parietal lobule

We also investigated the stimulus-induced modulations of power in the lower-frequency bands in terms of body-centered and gaze-centered lateralization. Figure $3, A$ and $B$, left panels, plots the scalp topography of the body-centered lateralization in the beta-band and the gaze-centered lateralization in the alpha band, respectively, in the same format as Figure 2, $A$ and $B$. The scalp topography is plotted for the sensory response interval; signifi- cant sensors are marked by dots. Beta-band activity $(18-30 \mathrm{~Hz})$ showed a trend of body-centered suppression of activity $(p<$ $0.10)$ at central sensors, closely overlapping with those showing the early body-centered power changes in the gamma band (Fig. $2)$. Alpha-band activity $(10 \mathrm{~Hz})$ showed no significant stimulusinduced body-centered power change ( $p>0.10$; data not shown) but demonstrated a clear gaze-centered suppression of power at central and posterior sensors $(p<0.05)$.

The middle panels of Figure $3, A$ and $B$, show the corresponding time-frequency representations of power, in an analysis pooled across hemispheres (see Materials and Methods). The stimulus artifact is indicated. The body-centered suppression of the beta band lasts until $\sim 600 \mathrm{~ms}$ after stimulus presentation and is followed by a rebound showing its peak halfway through the delay period (Fig. 3A, middle). As shown in Figure $3 B$, middle panel, gaze-centered suppression of the alpha band is maintained throughout the delay interval. Note that the panel also demonstrates significant gaze-centered suppression within the beta range $(p<0.05)$, at $\sim 20 \mathrm{~Hz}$. However, because of its frequency $(\sim 20 \mathrm{~Hz})$, its time course, as well as its scalp topography (data not shown), this power reduction is most likely best explained as a harmonic component of the dominant alpha band.

Source reconstructions of the various observations are presented in the right-hand panels of Figure 3. The body-centered contralateral suppression is localized at the postcentral gyrus, in primary somatosensory cortex, consistent with previous observations (Bauer et al., 2006; Haegens et al., 2010; van Ede et al., 2011). Note that this region also demonstrated body-centered increases in gamma-band power (Fig. 2A). Body-centered betaband suppression was further observed in the superior parietal lobe, whereas rebound effects were also seen in more distant extrastriate areas (Fig. 3A, bottom source reconstruction). Gaze- 


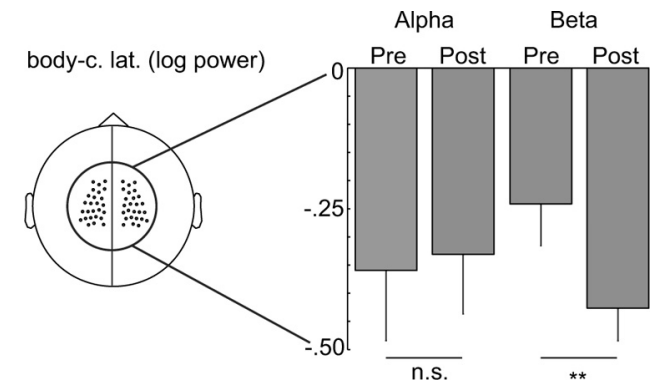

Figure 4. Alpha-and beta-band power at central sensors show body-centered lateralization in anticipation of the tactile stimulus. The negative values indicate that there is a power suppression on the contralateral side, compared with the ipsilateral side. Bar plots (with SE) represent body-centered alpha- and beta-band log power during the prestimulus ( $-300 \mathrm{~ms}$ ) and poststimulus response ( $400 \mathrm{~ms}$ ) intervals for the sensors marked in the left panel. All power values are significantly different from zero ( $t$ test, $p<0.05$ ). Asterisks indicate significant differences between prestimulus and poststimulus data ( ${ }^{* *} p<0.05$, paired $t$ test).

centered power modulations in the alpha band were found in parietal and parieto-occipital regions (Fig. 3B). Interestingly, the gazecentered alpha-band source and the body-centered beta-band source overlap at an area intermediate between somatosensory and visual cortices-the superior parietal lobule (SPL).

\section{Lower frequencies anticipate the stimulus}

Recall that our paradigm, by design, allowed subjects to anticipate the gross somatotopic body-centered coordinate (which hand receives the target), but not the retinotopic gaze-centered coordinate of the target (see Materials and Methods). For completeness, we therefore also compared the prestimulus modulations of power to the poststimulus modulations in the various frequency bands. We did not find any significant body-centered prestimulus increases in the gamma band $(p>0.05)$, consistent with previous reports (van Ede et al., 2011). For the lower frequencies - the beta and alpha bands - the results are shown in Figure 4, in log-power units, for the (central) sensors that showed body-centered selectivity in the beta band during the sensory response interval (Fig. 4, left). During the prestimulus period, both beta- and alpha-band power showed body-centered suppression at central sensors (i.e., less power at sensors contralateral than ipsilateral to the hand of stimulation). During the poststimulus period - the sensory response interval-power in the beta band is significantly further suppressed $\left({ }^{* *} p<0.05\right.$, paired $t$ test; consistent with Fig. $3 A$ ), while body-centered alpha-band suppression is maintained at a similar level as during the prestimulus period.

\section{Discussion}

We studied the spatial and temporal evolution of spectral power in various frequency bands during tactile remapping for saccades. Our results show that while gamma synchronization in S1 amplified the sensory response to the tactile stimulus relative to the body, replicating previous findings (Bauer et al., 2006; Lange et al., 2011), there was distinct gamma-band synchronization observed in IPS, reflecting the early remapping of the tactile stimulus during the sensory period relative to gaze. Power in the lower-frequency bands (alpha and beta) demonstrated body-centered suppression in S1, before the presentation of the tactile stimulus. These anticipatory effects were followed by a further suppression of beta-band (not alpha-band) power in both S1 and SPL after presentation of the stimulus. Power in the beta band then rebounded, not only in S1 and SPL, but also in extrastriate visual regions. In contrast, after presentation of the tactile stimulus, power in the alpha band maintained its body-centered selectivity at a constant level in S1, but showed gaze-centered suppression effects at occipital-parietal areas. We will next discuss the implications of our results.

\section{Sensory-induced remapping versus a prospective motor command}

A recent study investigated oscillatory activity during sensorimotor remapping in a delayed antisaccade task with visual targets (Van Der Werf et al., 2008). In this task, top-down executive control is required to transform the location of the visual stimulus into an eye movement to the opposite visual hemifield. In response to the stimulus, we observed a phasic broad-banded gamma-band response $(40-120 \mathrm{~Hz})$ in the posterior parietal cortex contralateral to the direction of the stimulus. Only after this sensory response had ended, the power increased in a gamma band in the PPC of the other hemisphere, which was contralateral to the planned saccade. In the present study, we also observed the sustained gamma-band response in the PPC contralateral to the direction of the saccade. However, the early gaze-centered response in PPC and other association regions was concurrently present with the sensory-induced somatotopic response in S1. In other words, gamma-band activity amplifies a representation relative to gaze in the PPC already during the ongoing sensory response, which argues in favor of a bottom-up remapping of a tactile stimulus in gaze-centered coordinates.

This bottom-up remapping mechanism is also reflected in the power modulation in the alpha band, which showed a gazecentered suppression in response to the tactile stimulus during the early sensory response interval. Previous studies have shown that alpha-band activity can be modulated by top-down or bottom-up visual spatial attention (Thut et al., 2006; Jensen and Mazaheri, 2010). Decreased oscillatory activity in the alpha band is further thought to reflect a state of enhanced cortical excitability (Romei et al., 2008). In terms of these interpretations, our data suggest that spatial attention is triggered (bottom-up) by tactile input, exciting the spatially corresponding regions in gazecentered association cortex at times similar to visual inputs, even when this must involve an interhemispheric transfer of information (Fig. 1B). During the late delay interval, alpha-band suppression increased in strength again, to support the mechanisms that are involved in preparation of the saccade (Van Der Werf et al., 2008).

Together, both the gamma and alpha frequency bands showed a temporal profile reflecting two sequential processes, a rather fast bottom-up sensory remapping followed by a memory process to maintain the goal of a later saccade. Previous studies have also provided evidence for the automatic remapping of tactile inputs, mediated by PPC (Azañón et al., 2010b; Pasalar et al., 2010). Using fMRI, Macaluso et al. (2002) found that tactile inputs are remapped to extrastriate visual areas, even when no saccades are involved in the task. Other studies have shown an influence of tactile stimuli on visual ERPs, taking into account hand position (Eimer and van Velzen, 2005) and an influence of hand posture on visuotactile integration (Schicke et al., 2009; Azañón et al., 2010a). Here, we show the spatiotemporal spectral signature of bottom-up remapping of tactile stimuli. Using connections between unisensory and multisensory cortices, the brain could generate spatially veridical predictions from one sensory modality to another, here encoding tactile targets in visual coordinates, which is consistent with suggestions by recent computational models (Pouget et al., 2002). 


\section{Sensory remapping routed by oscillations?}

The body-centered and gaze-centered lateralization of gammaband synchronization that both occur in response to the tactile stimulus overlap in time and partially in frequency, which may suggest that the map of S1 becomes transiently coupled to the retinotopic map in IPS by population synchronization. While structural connections alone cannot take into account the different eye-hand configurations, high-frequency synchronization could provide a mechanism for flexible functional coupling of regions, because it can change on a millisecond time scale (Fries, 2005). This notion is further supported by the observation that tactile stimuli induce power at higher gamma-band frequencies in the IPS than visual stimuli (Van Der Werf et al., 2008), in correspondence with the responses in their respective primary sensory areas ( $\mathrm{S} 1$ and primary visual cortex). This suggests that gamma-band synchronization in the IPS is not fixed, but might reflect a feedforward mechanism that adapts its frequency range to receive information encoded in a particular sensory region (somatosensory vs visual cortex, respectively). Supporting observations have been made in hippocampus, showing distinct gamma frequency bands for functional connections to different regions (Colgin et al., 2009). Also, communication of the PPC with different effector systems has been shown to involve different gamma-band frequencies (Van Der Werf et al., 2010). However, only invasive studies, which do not suffer from volume conduction problems, could directly test this hypothesis.

\section{Lower frequencies excite somatotopic and retinotopic cortex}

While high-frequency oscillations could provide the spectral channels for communication, low-frequency modulations have been proposed to play an important role in cross-modal attention (Lakatos et al., 2009). Indeed, beta-band activity was suppressed in body coordinates in central regions during the stimulus anticipation and further attenuated in S1 and SPL during the sensory response interval, consistent with a role for somatosensory processing and tactile attention (Bauer et al., 2006; van Ede et al., 2011). The SPL did not only show body-centered beta-band lateralization, but also showed power modulations in the gazecentered alpha band. This suggests that the SPL operates in multiple reference frames, which would be consistent with recent fMRI findings showing that SPL switches reference frames for different sensory contexts (Bernier and Grafton, 2010). Thus, body-centered beta and gaze-centered alpha modulations could preset the neuronal excitability of the SPL in both reference frames.

Body-centered beta-band lateralization rebounded after the sensory response interval, not only in S1 but also in extrastriate visual areas. Beta-band increases are typically observed in sensorimotor areas when no sensory, cognitive, or motor changes are expected (for review, see Engel and Fries, 2010). Our findings fit nicely with this general idea. We can only speculate about the role of the body-centered beta-band increases in visual areas. It is known that eye and hand position relative to the body modulate visual signals in a systematic manner, called gain fields (Andersen and Mountcastle, 1983). But whether the body-centered beta band supports the gain field mechanisms would require further studies.

Body-centered alpha-band suppression was observed in S1, in anticipation of the tactile stimulus. This suppression did not change after stimulus presentation. Thus, our results suggest two alpha-band sources: one body-centered in $\mathrm{S} 1$ and a gaze-centered source in the IPS. Also, the gaze-centered posterior alpha-band source has been shown to modulate in anticipation, when the location of a visual stimulus can be predicted (Thut et al., 2006). One could thus infer that anticipating a visuotactile event at the hand could lead to specific modulation of these sources, depending on eye-hand posture. That is, when the right hand is placed in the left visual field, there could be anticipatory suppression in left S1, and right IPS, together constituting a supramodal attention system that takes different eye-hand configurations into account.

In conclusion, the present study has shown that tactile targets are represented by activity in several frequency bands, operating in different reference frames. The temporal evolution of the gamma-band representations suggest that gaze-centered representations computed from somatotopic input are amplified by gamma-band synchronization in PPC early on during tactile remapping. In contrast to recent findings suggesting a sensory coding scheme in PPC (Bernier and Grafton, 2010), lateralization of posterior gamma-band activity was only congruent with gazecentered mapping for tactile stimuli at the population level. The early co-occurrence of gamma synchronization with different reference frames in the sensory (S1) and higher-order (PPC) map could imply that high-frequency oscillation serves as a mechanism to flexibly couple spatial maps of somatotopic and retinotopic formats, dependent on the relative positions of eyes and hand. This transient bottom-up induced representation, due to automatic remapping, is followed by a top-down enhanced representation that is sustained until the late delay. Low-frequency oscillations might support remapping by presetting the excitability of the involved neuronal substrates, based on eye-hand posture and anticipated stimulus locations.

\section{References}

Andersen RA, Cui H (2009) Intention, action planning, and decision making in parietal-frontal circuits. Neuron 63:568-583.

Andersen RA, Mountcastle VB (1983) The influence of the angle of gaze upon the excitability of the light-sensitive neurons of the posterior parietal cortex. J Neurosci 3:532-548.

Avillac M, Denève S, Olivier E, Pouget A, Duhamel JR (2005) Reference frames for representing visual and tactile locations in parietal cortex. Nat Neurosci 8:941-949.

Azañón E, Camacho K, Soto-Faraco S (2010a) Tactile remapping beyond space. Eur J Neurosci 31:1858-1867.

Azañón E, Longo MR, Soto-Faraco S, Haggard P (2010b) The posterior parietal cortex remaps touch into external space. Curr Biol 20:1304-1309.

Bastiaansen MC, Knösche TR (2000) Tangential derivative mapping of axial MEG applied to event-related desynchronization research. Clin Neurophysiol 111:1300-1305.

Bauer M, Oostenveld R, Peeters M, Fries P (2006) Tactile spatial attention enhances gamma-band activity in somatosensory cortex and reduces lowfrequency activity in parieto-occipital areas. J Neurosci 26:490-501.

Bernier PM, Grafton ST (2010) Human posterior parietal cortex flexibly determines reference frames for reaching based on sensory context. Neuron $68: 776-788$.

Chang SW, Snyder LH (2010) Idiosyncratic and systematic aspects of spatial representations in the macaque parietal cortex. Proc Natl Acad Sci U S A 107:7951-7956.

Chang SW, Papadimitriou C, Snyder LH (2009) Using a compound gain field to compute a reach plan. Neuron 64:744-755.

Colgin LL, Denninger T, Fyhn M, Hafting T, Bonnevie T, Jensen O, Moser MB, Moser EI (2009) Frequency of gamma oscillations routes flow of information in the hippocampus. Nature 462:353-357.

Eimer M, van Velzen J (2005) Spatial tuning of tactile attention modulates visual processing within hemifields: an ERP investigation of crossmodal attention. Exp Brain Res 166:402-410.

Engel AK, Fries P (2010) Beta-band oscillations_-signalling the status quo? Curr Opin Neurobiol 20:156-165.

Fries P (2005) A mechanism for cognitive dynamics: neuronal communication through neuronal coherence. Trends Cogn Sci 9:474-480.

Fries P, Womelsdorf T, Oostenveld R, Desimone R (2008) The effects of 
visual stimulation and selective visual attention on rhythmic neuronal synchronization in macaque area V4. J Neurosci 28:4823-4835.

Gross J, Kujala J, Hamalainen M, Timmermann L, Schnitzler A, Salmelin R (2001) Dynamic imaging of coherent sources: studying neural interactions in the human brain. Proc Natl Acad Sci U S A 98:694-699.

Haegens S, Osipova D, Oostenveld R, Jensen O (2010) Somatosensory working memory performance in humans depends on both engagement and disengagement of regions in a distributed network. Hum Brain Mapp 31:26-35.

Hämäläinen M, Hari R, Ilmoniemi RJ, Knuutila J, Lounasmaa OV (1993) Magnetoencephalography — theory, instrumentation, and applications to noninvasive studies of the working human brain. Rev Mod Phys 65: 413-497.

Jensen O, Mazaheri A (2010) Shaping functional architecture by oscillatory alpha activity: gating by inhibition. Front Hum Neurosci 4:186.

Lakatos P, O'Connell MN, Barczak A, Mills A, Javitt DC, Schroeder CE (2009) The leading sense: supramodal control of neurophysiological context by attention. Neuron 64:419-430.

Lange J, Oostenveld R, Fries P (2011) Perception of the touch-induced visual double-flash illusion correlates with changes of rhythmic neuronal activity in human visual and somatosensory areas. Neuroimage 54: 1395-1405.

Liljeström M, Kujala J, Jensen O, Salmelin R (2005) Neuromagnetic localization of rhythmic activity in the human brain: a comparison of three methods. Neuroimage 25:734-745.

Macaluso E, Frith CD, Driver J (2002) Crossmodal spatial influences of touch on extrastriate visual areas take current gaze direction into account. Neuron 34:647-658.

McGuire LM, Sabes PN (2009) Sensory transformations and the use of multiple reference frames for reach planning. Nat Neurosci 12:1056-1061.

Medendorp WP, Kramer GF, Jensen O, Oostenveld R, Schoffelen JM, Fries P (2007) Oscillatory activity in human parietal and occipital cortex shows hemispheric lateralization and memory effects in a delayed double-step saccade task. Cereb Cortex 17:2364-2374.

Mullette-Gillman OA, Cohen YE, Groh JM (2005) Eye-centered, headcentered, and complex coding of visual and auditory targets in the intraparietal sulcus. J Neurophysiol 94:2331-2352.

Mullette-Gillman OA, Cohen YE, Groh JM (2009) Motor-related signals in the intraparietal cortex encode locations in a hybrid, rather than eyecentered reference frame. Cereb Cortex 19:1761-1775.

Nolte G (2003) The magnetic lead field theorem in the quasi-static approximation and its use for magnetoencephalography forward calculation in realistic volume conductors. Phys Med Biol 48:3637-3652.

Pasalar S, Ro T, Beauchamp MS (2010) TMS of posterior parietal cortex disrupts visual tactile multisensory integration. Eur J Neurosci 31: 1783-1790.

Percival D, Walden A (1993) Spectral analysis for physical applications: multitaper and conventional univariate techniques. Cambridge, UK: Cambridge UP.

Pesaran B, Pezaris JS, Sahani M, Mitra PP, Andersen RA (2002) Temporal structure in neuronal activity during working memory in macaque parietal cortex. Nat Neurosci 5:805-811.

Pouget A, Deneve S, Duhamel JR (2002) A computational perspective on the neural basis of multisensory spatial representations. Nat Rev Neurosci 3:741-747.

Romei V, Brodbeck V, Michel C, Amedi A, Pascual-Leone A, Thut G (2008) Spontaneous fluctuations in posterior alpha-band EEG activity reflect variability in excitability of human visual areas. Cereb Cortex 18:20102018.

Russo GS, Bruce CJ (1994) Frontal eye field activity preceding aurally guided saccades. J Neurophysiol 71:1250-1253.

Schicke T, Bauer F, Röder B (2009) Interactions of different body parts in peripersonal space: how vision of the foot influences tactile perception at the hand. Exp Brain Res 192:703-715.

Stricanne B, Andersen RA, Mazzoni P (1996) Eye-centered, head-centered, and intermediate coding of remembered sound locations in area LIP. J Neurophysiol 76:2071-2076.

Thut G, Nietzel A, Brandt SA, Pascual-Leone A (2006) Alpha-band electroencephalographic activity over occipital cortex indexes visuospatial attention bias and predicts visual target detection. J Neurosci 26:9494-9502.

Van Der Werf J, Jensen O, Fries P, Medendorp WP (2008) Gamma-band activity in human posterior parietal cortex encodes the motor goal during delayed prosaccades and antisaccades. J Neurosci 28:8397-8405.

Van Der Werf J, Jensen O, Fries P, Medendorp WP (2010) Neuronal synchronization in human posterior parietal cortex during reach planning. J Neurosci 30:1402-1412.

van Ede F, de Lange F, Jensen O, Maris E (2011) Orienting attention to an upcoming tactile event involves a spatially and temporally specific modulation of sensorimotor alpha- and beta-band oscillations. J Neurosci 31:2016-2024.

Van Pelt S, Toni I, Diedrichsen J, Medendorp WP (2010) Repetition suppression dissociates spatial frames of reference in human saccade generation. J Neurophysiol 104:1239-1248.

Van Veen BD, van Drongelen W, Yuchtman M, Suzuki A (1997) Localization of brain electrical activity via linearly constraint minimum variance spatial filtering. IEEE Trans Biomed Eng 44:867-880.

Womelsdorf T, Fries P (2007) The role of neuronal synchronization in selective attention. Curr Opin Neurobiol 17:154-160. 